\author{
REVISTA DE CHIMIE \\ BUCURESTI ROMANIA \\ COLEGIUL EDITORIAL \\ Editor Sef \\ Petru FILIP \\ Institutul de Chimie Organics C.D. Nenitescu Bucuresti \\ Editor Sef Adjunct \\ Gheorghita J INESCU \\ Universitatea Politehnica Bucuresti
}

\author{
Alexandru T. BALABAN \\ Texas A\&M University at Galveston, SUA \\ IOn BOLOCAN \\ Universitatea Petrol Gaze Ploiesti \\ Miron Teodor CAPROIU \\ Institutul de Chimie Organica C.D. Nenitescu Bucuresti \\ Andrei F. DANET \\ Universitatea Bucuresti \\ Elena DIACU \\ Universitatea Politehnica Bucuresti \\ Gheorghe DUCA \\ Academia de Stiinte a Moldovei \\ Kamel EARAR \\ Universitatea Dunarea de J os Galati \\ Laurentiu FILIPESCU \\ Universitatea Politehnica Bucuresti \\ lonel HAIDUC \\ Academia Romana \\ Valeriu V.JINESCU \\ Universitatea Politehnica Bucuresti \\ J iri J aromir KLEMES \\ University of Pannonia Veszprem Ungaria \\ J ean-Marie LEHN \\ Universite Louis Pasteur, Strasbourg, Franta \\ Tudor LUPASCU \\ Academia de Stiinte a Moldovei \\ Ionel MANGALAGIU \\ Universitatea Alexandru Ioan Cuza lasi
}

Director General SYSCOM 18

IONANDRONACHE

Director SC BIBLIOTECA CHIMIEI SA

Carmen IOAN
MEMBRI

\author{
Gheorghe MARIA \\ Universitatea Politehnica Bucuresti \\ Gheorghe D. MATEESCU \\ Case Western Reserve University, Cleveland SUA \\ Lucian MOTIU \\ AGER Consulting SRL \\ Gheorghe NECHIFOR \\ Universitatea Politehnica Bucuresti \\ Aurel NECHITA \\ Universitatea Dunãrea de J os Galati \\ Luoana Florentina PASCU \\ INCDECOIND Bucuresti \\ Virgil PERCEC \\ University of Pennsylvania, Philadelphia SUA \\ Eli RUCKENSTEIN \\ The State University of New York at Buffalo, SUA \\ Sorin loan ROSCA \\ Universitatea Politehnica Bucuresti \\ Nicolae TOTIR \\ ICF I.G. Murgulescu Bucuresti \\ Paul VASILESCU \\ Universitatea Politehnica Bucuresti \\ Luminita VLADESCU \\ Universitatea Bucuresti \\ Maria ZAHARESCU \\ Academia Romana \\ Institutul de Chimie Fizica llie Murgulescu Bucuresti
}

Revista apare lunar

Copyright REVISTA DE CHIMIE

Raspunderea pentru datele publicate revine autorilor

Adresa redactiei: SC BIBLIOTECA CHIMIEI SAREVISTA DE CHIMIE

Calea Plevnei, Nr. 139B, Sector 6

Bucuresti 060011

Tel./Fax: (+ 40) 0213142447

www.revistadechimie.ro

\author{
Redactor Sef \\ Carmen IOAN \\ Tehnoredactare computerizata \\ Monica BALUTA
}

\author{
Factorul de impact pe anul 2015: 0,956 \\ Revista este recenzatã de: \\ CHEMICAL ABSTRACTS, CURRENT CONTENTS \\ ANALYTICAL ABSTRACTS \\ si indexata de \\ Institute for Scientific Information (ISI) si \\ Chemistry Citation Index
}




\section{REVISTA DE CHIMIE}

\author{
Bucharest-Romania \\ Chem. Abs.: RCBUAU 68 (1) (1-202) \\ ISSN 0034-7752 \\ ISSN CD-ROM: 2537-5733 \\ ISSN-L 1582-9049 \\ Vol. 68, no. 1, J anuary, 2017
}

$>$ Societatea de Chimie din România

$>$ SYSCOM 18 Bucuresti

$>$ SC Biblioteca Chimiei SA

\section{CONTENTS}

Rodica Elena Patescu, Claudia Maria Simonescu, C.Onose, T.L.Busuioc, Daiana Elena Pasarica, Carmen Deleanu

Simultaneous Removal of Lead and Nickel Ions from Aqueous Synthetic Solutions by Chitosan

Coated Cobalt Ferrite...

G.A.Popa, Daniela Florentina Popa (Enache), Dumitra Daniela Slave (Clej), I.D.Spiridon,

Cristina Monica Mirea, A.Ciocanea

Ultrafiltration of Aqueous Solutions of Food Dye in the Presence of Surfactants.

G.L.Gheorghievici, C.Trisca Rusu, Elena Voicila, I.M.Nafliu, Anca Maria Cimbru, Szidonia Katalin Tanczos

Titanium Dioxide for Biomedical Uses. I.The controlled production of nanoparticles by hydrothermal synthesis moderated by dimedone.

R.Hainarosie, V.Zainea, O.Ceachir, Mura Hainarosie, Catalina Pietrosanu, C.D.Stefanescu

The Use of Methylene Blue in Early Detection of the Vocal Fold Cancer..

Kasia Bloch, M.Nabialek. M.Szota

The Effect of Isothermal Annealing on the Structural and Magnetic Properties of the Bulk Amorphous Alloy:FeCoTiYB...18

M.Nabialek, P. Pietrusiewicz, M.Szota, M.M.Al Bakri Abdullah, A.V.Sandu

The Structure and Porosity of $\mathrm{Fe}_{62-\mathrm{x}} \mathrm{CO}_{10} \mathrm{~W}_{\mathrm{y}} \mathrm{Me}_{\mathrm{x}} \mathrm{Y}_{8} \mathrm{~B}_{20-\mathrm{y}}$ Alloys in the Amorphous and Crystalline States

(where:Me $=\mathrm{Mo}, \mathrm{Nb} ; \mathrm{x}=0,1,2 ; \mathrm{y}=0,1,2$ ).

Marilena Nicolae

Complex Systems of Distillation Columns Used in The Production of the Propylene Oxide.

Raluca Elena Dragomir, P.Rosca, T.J uganaru

Upgrading FCC LightCycle Oil.

D.R.Popoviciu, Ticuta Negreanu Pirjol, L.S.Miclaus

Phytotoxic Effectand Bioaccumulation of Chromium in White Mustard (Sinapis alba L.) Seedlings

M.Tampa, Ilinca Nicolae, Corina Daniela Ene, Isabela Sarbu, Clara Matei, Simona Roxana Georgescu

Vitamin C and Thiobarbituric Acid Reactive Substances (TBARS) in Psoriasis Vulgaris Related

to Psoriasis Area Severity Index (PASI)

Catalina Iulia Saveanu, Oana Dragos, V.Danila, Corina Cheptea

Ultrastructural Analysis by Scanning Electron Microscopy of Dental Structures Conditioning with

Orthophosphoric Acid and ER.CR:YSGG Laser Irradiation...

Daniela Radulescu, Andra Elena Balcangiu Stroescu, C.Pricop, B.Geavlete, Carolina Negrei, O.Bratu,

O.Ginghina, Ileana Adela Vacaroiu

Vitamin K Influence on Cardiovascular Mortality in Chronic Hemodialysed Patients.

V.G.Vasilescu, Elisabeta Vasilescu, D.Dima, T.L.Ciocan, A.Semenescu, B.Florea, Ileana Mariana Mates,

Oana Roxana Chivu

Contributions on Setting the Optimal Regime of Antibacterial Deposition on The Surface of the Oral Implant

of Ti10Zr Bio-alloy...

Daniela Simina Stefan, Nicoleta Neacsu, Irina Pincovschi, M.Stefan

Water Quality and Self-purification Capacity Assessment of Snagov Lake...

E.Tudor, T.Cioroianu, Carmen Sirbu, M.Dumitru, Adriana Grigore, Lavinia Parvan

Fertilizer for the Treatment of Iron Chlorosis.Physico-chemical and Agro-chemical Properties.

Daniela Cirtina, Camelia Capatina

Quality Issues Regarding the Watercourses from Middle Basin of J iu River.

V.M.Dumitrascu, Lidia Benea

Improving the Corrosion Behaviour of 6061 Aluminium Alloy by Controlled Anodic Formed Oxide Layer.

M.Matei, I.Sandu, M.L.Birsa, Laura Gabriela Sarbu, L.Simion

New 4-(4-Hydroxyaryl)-5-Methyl-1,3-Dithiol-2-ylidene Derivatives.

Vasilica Vasile, Alina Dima, Mihaela Ion

Monitoring of the Inorganic Pollutants in BuiltIndoor Environment.

Anisia Iuliana Alexa, Alina Cantemir, A.Ciobica, I.Gardikiotis, Iulia Antioch, C.Honceriu,

Carmen Lacramioara Zamfir, V.Poroch

Preliminary Data Regarding Decreased Catalase Specific Activity in the Tears as a Result of Environmental -stress. 
Oana Tanculescu, A.Doloca, Raluca Maria Vieriu, Florentina Mocanu, Gabriela Ifteni, Anca Vitalariu, Sorina Solomon, Nicoleta Ioanid, Gianina Iovan

Load-Bearing Capacity of Direct Inlay-Retained,Fibre-reinforced Composite Fixed Partial Dentures with

Different Cross-Sectional Pontic Design

Song II Kong, Danuta Matei, Diana Cursaru, V.Matei, D.Ciuparu

Characterization and Stability of Heteropolyacid Catalysts Supported on MCM-41 Materials Synthesized by

Ultrasonic Irradiation.....

Gina Botnariu, Norina Forna, Alina Popa, Raluca Popescu, Alina Onofriescu, D.Cioloca,

Cristina Lacatusu, B.Mihai

Correlation of Glycemic Control Parameters in Non-Diabetic Person with Cardiovascular Risk Scores-Results

from a Cross-Sectional Study.......

Zoia Apostolescu, Oana Roxana Chivu, A.Semenescu, C.Babis, C.Amza, G.lacobescu,

Maria Alina Gligor, D.Nitoi, G. M. Adir

The Impacts of Industrial Processing of Oil on Soil Quality...

I.V.Asaftei, N.C.Lungu, L.M.Birsa, I.G.Sandu, Laura Gabriela Sarbu, Maria Ignat

Performance of Ag-HZSM-5 Zeolite Catalysts in n-heptane Conversion...

Sinziana Venera Morarita, Ana Maria Hossu, Andreea Antonia Georgescu

The Study of Analysed Biochemical Parameters on Fruits of Prunus Cerasifera Ehrh. Biotypes...

Alexandrina Muntean, Anca Stefania Mesaros, Anca Porumb, Stanca Cuc, Marioara Moldovan,

Adriana Balan

Enamel Apperance after Orthodontic Attachment Removal. In vitro SEM analysis.

Adriana Marinoiu, Elena Carcadea, Claudia Cobzaru, Corina Cernatescu

Numerical Approach for Catalytic Conversion of $\mathrm{CO}_{2}$ to Methane over Nickel Base Catalysts.

S.Andrian, Gianina lovan, Angela Cristina Ghiorghe, Galina Pancu, A.Georgescu,

Dana Nicoleta Antonescu, Simona Stoleriu

Study Regarding the Effect of Some Remineralizing Products Used in the Tratment of Erosion Induced

by SportDrinks.

Mihaela Neagu, Daniela Roxana Popovici, Cristina Maria Dusescu, Catalina Calin

Adsorption of $\mathrm{p}$-Cresol by Mesoporous Activated Carbon. I.Kinetics studies and data modeling.

A.Erdem, H.Genc, N.Sen, R.Kilincarslan, E.Erdem

The Synthesis and Reactions of Novel Pyrazole Derivatives by 4-phenylcarbonyl-5-phenyl-2,3-dihydro-2,3-furandione

Reacted with Some Hydrazones.

P.Chirita, I.V.Asaftei, I.Sandu, Laura Gabriela Sarbu, V.V.Lupu

Mesoionic 4-(2-Dialkylamino-1,3-dithiol-2-ylium-4-yl)phenolates..

M.G.Dolis, Cristina Simeanu, A.Usturoi, D.Simeanu

Research Regarding Chemical Composition and the Digestibility of the Mulberry Leaves from Eforie Variety...

Irina Ciotlaus, Lucia Gansca, Adriana Maria Andreica, I.Oprean

A practical Synthesis of (Z)- and (E)-8-Dodecene-1-yl Acetate, Components of Lepidoptera

InsectSex Pheromones.

Luminita Lazar, Cristina Ioana Bica, Krisztina Martha, Mariana Pacurar, E.Bud, Ana Petra Lazar,

Lilla Lorinczi

The Use of Polymerase Chain Reaction (PCR) for Identifying Periodontopathogenic Bacteria- Therapeutic

Implications in Periodontal Disease.

Letitia Doina Duceac, Cristina Elena Dobre, Ioana Pavaleanu, Gabriela Calin, Simona Nichitus,

Daniela Damir

Diseases Prevention by Water Defluoridation Using Hydrotalcites as Decontaminant Materials.

B.H.Serb, Alina Ormenisan, Ancuta Pleseru, V.Preluca, Mihaela Cernusca Mitariu, Corina Roman Filip,

H.M.Barbu, S.Cernusca Mitariu

Pharmacodynamic Modulation of Motility by Glycine Administartion in Dugesia dorotocephala.

Oana Roxana Chivu, A.Semenescu, C.Babis, C.Amza, G.Iacobescu, Zoia Apostolescu,

V.Petrescu, G.M.Adir

The Impact of the Industrial Processing of Oil on the Rainfall Water Quality...

Adriana Maria Andreica, Lucia Gansca, Irina Ciotlaus, I. Oprean

Convenient Synthesis of (Z)-7-and (E)-9-dodecene-1-yl Acetate, Components

of Some Lepidoptera InsectSex Pheromone...

Gabriela Balan, Camelia Ana Grigore, C.C. Budacu, Alina Calin, M. Constantin, Catalina Mihaela Luca

Antisepsis, Disinfection Sterilization - Methods Used in Dentistry...

A. Almasi, Anca Porumb, Angela Codruta Podariu, Liana Todor, S. Al. Tofan, Ramona Amina Popovici

The Effects of Nanofillers on Composite Materials Mechanical Properties.

Al. Patrascu, Liliana Savin, Olivera Lupescu, D. Mihailescu, Dana Nicoleta Mihai,

M. Neculaes, V. Grigorescu, Carmen Greierosu, P. Botez

Multifocal Osteonecrosis Glucocorticoid Induced. 


\section{REVISTA DE CHIMIE}

\author{
Bucuresti-Romania \\ Chem. Abs.: RCBUAU 68 (1) (1-202) \\ ISSN 0034-7752 \\ ISSN CD-ROM: 2537-5733 \\ ISSN-L 1582-9049 \\ Vol. 68, nr. 1, ianuarie, 2017
}

$>$ Societatea de Chimie din România

$>$ SYSCOM 18 Bucuresti

$>$ SC Biblioteca Chimiei SA

\section{CUPRINS}

\section{Rodica Elena Patescu, Claudia Maria Simonescu, C.Onose, T.L.Busuioc, Daiana Elena Pasarica,}

Carmen Deleanu

Indepartarea simultana a ionilor de plumb si nichel din solutii apoase utilizand ferita de cobalt acoperita cu chitosan......1 G.A.Popa, Daniela Florentina Popa (Enache), Dumitra Daniela, Slave (Clej), I.D.Spiridon, Cristina Monica Mirea, A.Ciocanea

Ultrafiltrarea solutiilor apoase cu decoloranti alimentari in prezenta surfactantilor

G.L.Gheorghievici, C.Trisca Rusu, Elena Voicila, I.M.Nafliu, Anca, Maria Cimbru, Szidonia Katalin Tanczos

Dioxid de titan pentru uz biomedial. I. Productia controlatra a nanoparticulelor

prin sinteza moderata de dimedona.

R.Hainarosie, V.Zainea, O.Ceachir, Mura Hainarosie, Catalina Pietrosanu, C.D.Stefanescu

Utilizarea solutiei de albastru de metil in detectarea precoce a neoplasmelor corzii vocale.

Kasia Bloch, M.Nabialek, M.Szota

Efectul de calire izoterma asupra proprietatilor structurale si magnetice ale aliajului amorf bulk: FeCoTiYB.

M. Nabialek, P. Pietrusiewicz, M.Szota, M.M.Al Bakri Abdullah, A.V. Sandu

Structura si porozitatea aliajului $\mathrm{Fe}_{22-x} \mathrm{Co}_{10} \mathrm{~W}_{y} \mathrm{Me}_{x} \mathrm{Y}_{8} \mathrm{~B}_{20-y}$ in stare amorfa si cristalina

(unde: $\mathrm{Me}=\mathrm{Mo}, \mathrm{Nb} ; \mathrm{x}=0,1,2 ; \mathrm{y}=0,1,2$ ).

Marilena Nicolae

Sisteme de coloane complexe utilizate la fabricarea propenoxidului. .22

Raluca Elena Dragomir, P.Rosca, T.J uganaru

Imbunatatirea procesului de hidrocracare a unui amestec de motorine.

D.R.Popoviciu, Ticuta Negreanu Pirjol, L.S.Miclaus

Efectul fitotoxic si bioacumularea cromului in semintele de mustar alb...

M.Tampa, Ilinca Nicolae, Corina Daniela Ene, Isabela Sarbu, Clara Matei, Simona Roxana Georgescu

Vitamina C si acidul tiobarbituric substante reactive in Psoriasis Vulgaris corelat cu indexul

de severitate al domeniului psoriasis.

Catalina Iulia Saveanu, Oana Dragos, V.Danila, Corina Cheptea

Analiza ultrastructurala utilizand microscopia electronica prin scanare a structurii dentinare conditionata cu acid

ortofosforic si prin iradiere cu ER.CR:YSGG Laser.

Daniela Radulescu, Andra Elena Balcangiu Stroescu, C.Pricop, B.Geavlete, Carolina Negrei, O.Bratu,

O.Ginghina, Ileana Adela Vacaroiu

Asocierea vitaminei $\mathrm{K}$ cu mortalitatea de cauza cardiovasculara la pacientii hemodializati cronic.

V.G.Vasilescu, Elisabeta Vasilescu, D.Dima, T.L.Ciocan, A.Semenescu, B.Florea, Ileana Mariana Mates,

Oana Roxana Chivu

Contributii privind stabilirea regimului optim de depunere antibacteriana pe suprafata implantului

oral din bioaliaj Ti10Zr.......

Daniela Simina Stefan, Nicoleta Neacsu, Irina Pincovschi, M.Stefan

Calitatea apei si capacitatea de autopurificare a Lacului Snagov.

E.Tudor, T.Cioroianu, Carmen Sirbu, M.Dumitru, Adriana Grigore, Lavinia Parvan

Fertilizanti pentru tratarea clorozei ferice. Proprietati fizico-chimice si agrochimice.

Daniela Cirtina, Camelia Capatina

Aspecte privind caracterizarea calitatii unor cursuri de apa din bazinul hidrografic mijlociu al raului j iu.

V.M.Dumitrascu, Lidia Benea

Imbunatatirea comportamentului la coroziune a aliajului Al6061 prin formarea controlata anodic

astratului de oxid

M.Matei, I.Sandu, M.L.Birsa, Laura Gabriela Sarbu, L.Simion

Noi derivati de 4-(4-hidroxiaril)-1,3-ditiol-2-iliden..

Vasilica Vasile, Alina Dima, Mihaela Ion

Monitorizarea poluantilor anorganici in mediul interior construit 
Anisia Iuliana Alexa, Alina Cantemir, A.Ciobica, I.Gardikiotis, Iulia Antioch, C.Honceriu,

Carmen Lacramioara Zamfir, V.Poroch

Date preliminare privind activitatea specifica scazuta a catalazei din lacrimi ca urmare

a stresului din mediul inconjurator...

Oana Tanculescu, A.Doloca, Raluca Maria Vieriu, Florentina Mocanu, Gabriela Ifteni, Anca Vitalariu,

Sorina Solomon, Nicoleta Ioanid, Gianina Iovan

Capacitatea portanta a compozitelor ranforsate cu fibre pentru protezele fixe partiale......

Song II Kong, Danuta Matei, Diana Cursaru, V.Matei, D.Ciuparu

Caracterizarea si stabilitatea catalizatorilor heteropoliacid suportati pe materiale MCM-41 sintetizati

prin iradiere ultrasonica.

Gina Botnariu, Norina Forna, Alina Popa, Raluca Popescu, Alina Onofriescu, D.Cioloca,

Cristina Lacatusu, B.Mihai

Evaluarea corelatiei dintre parametrii principali ai controlului glicemic si scorurile de risc

cardiovascular la persoanele nondiabetice.

Zoia Apostolescu, Oana Roxana Chivu, A.Semenescu, C.Babis, C.Amza, G.lacobescu,

Maria Alina Gligor, D.Nitoi, G. M. Adir

Impactul activitatii de procesare industriala a petrolului asupra calitatii solului.

I.V.Asaftei, N.C.Lungu, L.M.Birsa, I.G.Sandu, Laura Gabriela Sarbu, Maria Ignat

Performanta catalizatorului zeolitic Ag-HZSM-5 in conversia n-heptanului..

Sinziana Venera Morarita, Ana Maria Hossu, Andreea Antonia Georgescu

Studiul parametrilor biochimici ai fructelor de corcodus.....

Alexandrina Muntean, Anca Stefania Mesaros, Anca Porumb, Stanca Cuc, Marioara Moldovan,

Adriana Balan

Evaluarea aspectului smaltului dentar dupa indepartarea atasamentelor ortodontice. Analiza SEM in vitro. 125

Adriana Marinoiu, Elena Carcadea, Claudia Cobzaru, Corina Cernatescu

Model matematic pentru conversia catalitica a $\mathrm{CO}_{2}$ la metan pe catalizatori pe baza de nichel..

S.Andrian, Gianina lovan, Angela Cristina Ghiorghe, Galina Pancu, A.Georgescu,

Dana Nicoleta Antonescu, Simona Stoleriu

Studiu privind actiunea unor produse de remineralizare utilizate in tratamentul eroziunilor induse

de bauturile pentru sportivi.

Mihaela Neagu, Daniela Roxana Popovici, Cristina Maria Dusescu, Catalina Calin

Adsorbtia paracrezolului pe carbune mezoporos activat. I. Studii cinetice si date de modelare.

A.Erdem, H.Genc, N.Sen, R.Kilincarslan, E.Erdem

Sinteza si reactille unor noi derivati ai pirazolului reactionati cu unele hidrazone.

P.Chirita, I.V.Asaftei, I.Sandu, Laura Gabriela Sarbu, V.V.Lupu

Fenolati mezoionici de 4-[2--dialchilamino-1,3-ditiol-2-iliu-4-il]......

M.G.Dolis, Cristina Simeanu, A.Usturoi, D.Simeanu

Cercetari privind compozitia chimica si digestibilitatea frunzelor de dud din varietatea Eforie.

Irina Ciotlaus, Lucia Gansca, Adriana Maria Andreica, I.Oprean

Sinteza (Z)- si (E)-8 dodecen-1-il-acetat componente ale feromonilor sexuali ai insectelor din ordinul Lepidoptera......157

Luminita Lazar, Cristina Ioana Bica, Krisztina Martha, Mariana Pacurar, E.Bud,

Ana Petra Lazar, Lilla Lorinczi

Utilizarea polimerazei reactive pentru identificarea bacteriei periodontopatogenice -

implicatii terapeutice in afectiunile periodontale..

Letitia Doina Duceac, Cristina Elena Dobre, Ioana Pavaleanu, Gabriela Calin, Simona Nichitus,

Daniela Damir

Prevenirea afectiunilor prin deflorurarea apei utilizand hidrotalcite ca materiale decontaminante.

B.H.Serb, Alina Ormenisan, Ancuta Pleseru, V.Preluca, Mihaela Cernusca Mitariu, Corina Roman Filip,

H.M.Barbu, S.Cernusca Mitariu

Modularea farmacodinamica a motilitatii prin administrarea de glicina in Dugesia dorotocephala..

Oana Roxana Chivu, A.Semenescu, C.Babis, C.Amza, G.Iacobescu, Zoia Apostolescu,

V.Petrescu, G.M.Adir

Impactul activitatii de procesare industraiala a petrolului asupra calitatii apei de precipitatii..

Adriana Maria Andreica, Lucia Gansca, Irina Ciotlaus, I. Oprean

Sinteza(Z)-7- si(E)-9- dodecen1-il-acetat componente ale feromonilor sexuali ai insectelor

din ordinul Lepidoptera.

Gabriela Balan, Camelia Ana Grigore, C.C. Budacu, Alina Calin, M. Constantin, Catalina Mihaela Luca

Antisepsie, dezinfectie-metode de sterilizare utilizate in stomatologie...

A. Almasi, Anca Porumb, Angela Codruta Podariu, Liana Todor, S. Al. Tofan, Ramona Amina Popovici

Efectele nanofilerilor asupra proprietatilor mecanice ale materialelor compozite.

Al. Patrascu, Liliana Savin, Olivera Lupescu, D. Mihailescu, Dana Nicoleta Mihai,

M. Neculaes, V. Grigorescu, Carmen Greierosu, P. Botez

Osteonecroza multifocala indusa de glucocorticoizi... 


\section{REVISTA DE CHIMIE SI}

REVISTA MATERIALE PLASTICE POT FI ACCESATE GRATUIT LA URMATOARELE ADRESE:

http://www.revistadechimie.ro/ http://www.revmaterialeplastice.ro/

\section{FREE WEB ACCESS FOR REVISTA DE CHIMIE AND} MATERIALE PLASTICE

http://www.revistadechimie.ro/ http://www.revmaterialeplastice.ro/ 